\title{
User Friendliness of a Wearable Visual Behavior Monitor for Cataract and Refractive Surgery
}

\author{
Bojan Pajic 1,2,3,4,*(D), Pavel Zakharov ${ }^{5}$, Brigitte Pajic-Eggspuehler ${ }^{1}$ and Zeljka Cvejic ${ }^{2}$ (D) \\ 1 Eye Clinic Orasis, Swiss Eye Research Foundation, Titlisstrasse 44, 5734 Reinach AG, Switzerland; \\ brigitte.pajic@orasis.ch \\ 2 Department of Physics, Faculty of Sciences, University of Novi Sad, Trg Dositeja Obradovica 4, \\ 21000 Novi Sad, Serbia; zeljka.cvejic@df.uns.ac.rs \\ 3 Division of Ophthalmology, Department of Clinical Neurosciences, Geneva University Hospitals, \\ 1205 Geneva, Switzerland \\ 4 Faculty of Medicine of the Military Medical Academy, University of Defense, 11000 Belgrade, Serbia \\ 5 Vivior AG, Technoparkstrasse 1, 8005 Zürich, Switzerland; pavel.zakharov@vivior.com \\ * Correspondence: bpajic@datacomm.ch; Tel.: +41-62-765-6080
}

Received: 23 December 2019; Accepted: 17 March 2020; Published: 24 March 2020

\begin{abstract}
A prospective feasibility study was conducted to determine whether a new wearable device, the Visual Behavior Monitor (VBM), was easy to use and did not present any difficulties with the daily activities of patients. Patients for cataract surgery and refractive lens exchange were randomly selected and screened for inclusion in the study. A total of 129 patients were included in the study as part of a multicenter study. All measurements were performed before surgery. Upon inclusion, patients were trained to wear the device, instructed to wear it for a minimum of $36 \mathrm{~h}$, and were scheduled to return in one week. The VBM measures the distance at which patients' visual activities are performed, the level of illumination, and head translational and rotational movements along the three axes. On the follow-up visit, patients completed a questionnaire about their experience in wearing the device. All patients underwent standard diagnostic testing, with their cataract grade determined by the Lens Opacities Classification System (LOCS) classification. Results indicate that $87 \%$ of patients felt comfortable using the wearable device while $8 \%$ of patients responded as not feeling comfortable ( $5 \%$ of patients did not respond to the question). In addition, $91 \%$ of patients found it easy to attach the wearable to the magnetic clip while $4 \%$ of patients did not find it easy, and $5 \%$ of patients did not respond. Overall, patients found the device easy to use, with most reporting that the device was not intrusive.
\end{abstract}

Keywords: cataract surgery; refractive surgery; visual behavior monitor

\section{Introduction}

Cataract surgery has become the most common operation worldwide. In the United States alone, 3.6 million cataract surgeries are performed each year. This corresponds to a cataract surgery rate (CSR) of 11,000 per 1 million inhabitants. In economically well-developed countries such as the United States, Europe, Australia, or Japan, the CSR is between 4000 and 11,000 [1,2]. Yet, refractive outcomes, and patient satisfaction, following cataract surgery remain a key challenge for ophthalmologists today, particularly when it involves a presbyopia-correcting intraocular lens (IOL) [3].

In an era where we strive to improve on our outcomes by using increasingly sophisticated biometry and IOL power calculations, it is important not to lose sight of the impact of residual or increased refractive errors that may not be apparent using the spherical equivalent or viewing the components of the refractive error in isolation on the patient's vision. We should use the tools that facilitate surgeons to assess and manage the patient's refractive error in its entirety. 
Approaching the intended post-operative spherical equivalent, however, often does not achieve spectacle independence. Uncorrected residual spherocylindrical refractive errors appear to have far greater adverse effects on unaided visual acuity than may be evident using a spherical equivalent or the individual sphere and cylinder. If the correction of presbyopia is desired, there are three options available for IOLs in the course of cataract surgery. One is the installation of a monovision where the dominant eye is set for distance and the non-dominant eye for near. A difference of up to $2 \mathrm{D}$ is accepted by the patient. However, before proceeding to surgery, it is essential to try wearing contact lenses to see if the correction is tolerated [4,5]. Most patients reported the surgery met their expectations for decreased dependence on spectacles (93\%) [6]. The extended-depth-of-focus-IOL (EDOF-IOL) provides an extended depth-of-field range. Actually, it is a bifocal IOL with a low near-addition of 1.5-2.0 D. This results in excellent distance and intermediate correction without the disadvantages of a multifocal IOL. However, this procedure does not completely eliminate the need for spectacles $[5,7]$. The third possibility of correction is the implantation of a trifocal IOL. One is located in the distance and receives $50 \%$ of the incident light, the second is at $30-40 \mathrm{~cm}$ and receives $30 \%$ of the light, and the third is at about $60 \mathrm{~cm}$ and receives $20 \%$ of the light. The strength of trifocal IOLs is that they are glasses-free. The weakness is that fixed distances are given, which requires some getting used to, and that the intermediate part is the weakest. As only a part of the light used for image generation is sharp, there is a loss of contrast, which is noticeable. In addition, undesirable optical side effects in the form of so-called halos or glare occur, which impair vision, especially at twilight and at night $[5,8]$. There is no ideal correction for all distances, but through objective measurement using the Visual Behavior Monitor (VBM) regarding distance measurement, ambient lighting and head posture will potentially determine a much more accurate setting and support the choice of IOL. Whatever additional technique is used, an important measure of success is the difference between the intended and the actual postoperative refractive outcome. It is necessary to have a method that is sensitive to discrepancies between the intended and the actual outcome.

Hence, to minimize potential side effects and improve patient outcomes, IOLs must be chosen with the visual needs of a patient in mind. One such method to assess a patient's individual visual needs is to determine patient expectations and visual behavior pre-operatively, to improve post-operative outcomes.

Visual behavior monitors are developed through various technological approaches, which should be as precise as possible but also cost-effective. Various studies use active lighting based on infrared LEDs. For example, one study [9] proposes a system that uses 3D vision techniques to estimate and track the 3D line of sight of a person with multiple cameras. The method is based on a simplified eye model and uses the Purkinje images of an infrared light source to determine the eye position. This information is used to estimate the line of sight. In a technological development, a system with active infrared LED illumination and one camera is implemented. Due to the LED illumination, the method can easily find the eyes and the system uses this information to locate the remaining facial features [10]. The authors suggest estimating the local direction of gaze analytically based on the pupil position. Almost all active systems described in the literature have been tested in simulated environments, but not in real ones. A moving person presents new challenges such as variable lighting, changing background, and vibrations that have to be considered in real systems. In a further work [11], an industrial prototype called Copilot is presented whose application tends to take into account the real situation. This system uses an infrared LED illumination to find the eyes. It uses a simple subtraction procedure to find the eyes and calculates only one validated parameter, the percentage eye closure (PERCLOS). This system currently works in low-light conditions [12]. All of these technologies, including ours, tend to collect even more precise data and reflect reality. Further improvements can be expected in the future by combining different technologies. Many technologies are developed in such a way that they are easy to handle and can be spread further. For example, the investigation tools are built into smartphones [13]. This is the way we initially went. However, a further simplification of the operation has become necessary after initial experiences. This led to today's VBM, which does not 
require any technical knowledge of the patient. Due to the simplicity, the measurement results are much more precise and reflect reality much better.

Results from a literature review suggest that there is a greater utility of using measurable patient reported outcomes such as functioning, satisfaction, and quality of life as opposed to clinical outcomes when considering the benefits of cataract surgery $[1,14]$.

Psychometric tests in the form of cataract surgery outcome questionnaires have been developed, identifying the limitations of performing daily tasks due to an individual's vision, a trait known as visual functioning $[15,16]$. Analyzing the visual behavior of cataract patients is extremely important for defining the visual strategy adopted in the patient's care.

McAlinden et al. [16] studied the responsiveness of several questionnaires in measuring patient outcomes. The investigation revealed that "Catquest-9SF," a Rasch modified 9 item questionnaire originally developed in Sweden [17], was the most responsive as measured by an effect size (ES) statistic of 1.45 (95\% CI, 1.22-1.67). Although the "Catquest-9SF" includes a measure of visual functioning, a questionnaire known as the "Visual Disability Assessment (VDA)" (ES, 1.09; 95\% CI, 0.86-1.32) also includes a measure of mobility of the patient, which may be attractive for patients and clinicians. Finally, the VBM measurement supports the solution finding for a planned surgery.

Morlock et al. [18] developed a Patient-Reported Spectacle Independence Questionnaire (PRSIQ) to assess spectacle independence following cataract surgery. The questionnaire was developed using conventional qualitative and modern measurement theory methods, and evidence was garnered for the use of PRSIQ as a measure of spectacle independence.

While the use of questionnaires and visual assessments in measuring patient outcomes are effective, there are limitations regarding the subjectivity of the results. Current psychometric measures may be subject to systematic error such as recall bias and could cause judgment errors resulting in patient discontentment with surgical outcomes $[19,20]$. Visual information based on patient behavior can be harnessed using technological tools that perform eye tracking, analyzing visual behavior under various circumstances. Eye tracking technologies can be used to attain and process objective information of a patient's everyday activities, crucial for selecting an appropriate IOL.

Integrating technology that collects objective visual behavior data into pre-operative patient assessments has shown to improve the current understanding of patient behavioral gaze. The data generated could aid clinicians to provide a highly individual evaluation of a patient's visual needs, including distance estimates of the patient's sight, and determine the right course of action to improve post-operative patient outcomes and patient satisfaction.

To date, most approaches have focused on diagnostic testing in a clinical setting. In-clinic measurements cannot fully replicate a patient's daily visual needs. The Visual Behavior Monitor (VBM, Vivior AG, Zurich, Switzerland) is a wearable device to be attached on the spectacles of a patient and perform continuous measurements as he or she goes about their daily activities. The challenge of unassisted monitoring in outpatient settings is ensuring correct use of the monitoring system and reliability of the data. This is especially the case for the senior cataract patients with compromised visual function due to age-related cataract and/or presbyopia. While aiming to develop a compact and non-obtrusive system, it is essential to ensure that the reduced form-factor of the device does not impede the handling of the device by the elderly population. Another challenge is to design the user interfaces of the system in a way that can be correctly interpreted by patients with different levels of exposure to computer and information technologies. Most of the recent progress in the information technologies has been pushed forward generally by the young generations of earlier adopters and, thus, interfaces are shaped for and by this population group, who have enough time and interest to work with new technologies. It is critically important to redesign the user experience from scratch with wearable systems for all population groups.

Inadequately designed systems ignoring the specifics of the population groups might not only be inefficient in delivering required objective information, but also lead to reduced patient satisfaction due to frustration from handling the device. This is aggravated by the patient awareness that the outcome 
of the surgery might depend on his or her ability to handle the system. With all the above in mind, the system should be comfortable for use by the patients of various age groups, educational backgrounds with different levels of exposure to information technology, and possibly with compromised vision.

The present study was initiated to understand the feasibility of the non-intrusive monitoring of visual behavior and VBM handling by, particularly, a cataract-age population without supervision of a healthcare professional (HCP). The study is designed to evaluate the ease of use, as well as patient perception of wearing the device. This can potentially answer the question of whether patients are willing to use and able to handle the wearable device mounted on the spectacles and are comfortable to use it in order to perform the required measurements.

\section{Materials and Methods}

Patients presenting for cataract surgery or refractive lens exchange were randomly selected to take part in this feasibility study to evaluate the VBM. Patients who met the inclusion criteria were recruited consecutively for the study. Upon enrolment, patients completed a modified Cat-Quest 9 questionnaire. For German-speaking sites, the questionnaire was modified to improve readability in German. Patients were trained and fitted with the VBM and instructed to wear the device for at least $36 \mathrm{~h}$. Patients then returned a week later to complete a questionnaire on the user friendliness of the device and schedule surgery. In our study, we used a modified CATQ-9 questionnaire, which refers to the patient's ability to perform certain tasks. The questionnaire consists of 9 questions, with each question being rated on a scale of 1-4-from 1 for "no problems" to 4 for "very big problems." In general, it is asked whether the patient has problems with vision in everyday life and how satisfied the patient is with his or her vision. In particular, the questions ask how well the patient sees on the computer, how well he/she recognizes faces, how well he/she recognizes prices when shopping, how well he/she walks on uneven ground, how he/she watches television with subtitles, and what is his/her favorite pastime and hobbies. This covers a large part of daily life.

The following inclusion criteria are applied: Age over 18 years old, participants considering IOL procedures, participants considering refractive laser procedures, and participants willing and able to participate. As exclusion criteria, we have defined the following points for the study: Unwillingness to participate, manifestation of macular disease based on OCT(Optical Coherence Tomography) /fundus photography, corneal scar or sign of corneal decompensation, standard surgery exclusion criteria, inability to make all postoperative visits, history or manifestation of other pre-existing ocular conditions and comorbidity (amblyopia, monophthalmia), other predisposing sight-threatening ocular conditions (uveitis, diabetic retinopathy, ARMD, macular dystrophy, retinal detachment, neuro-ophthalmic disease, abnormal pupils [deformation] and iris), history of corneal or retinal surgery, evidence of iris or chorioretinal neovascularization, history of anterior segment pathology (aniridia), any scar from corneal endothelium damage (chemical burn, herpetic keratitis, cornea guttata), any medical history contraindicative of standard cataract surgery including those taking medications known to potentially complicate cataract surgery (e.g., $\alpha 1$ a-selective alpha blocker), and participating in another clinical trial.

The primary endpoint of the study is testing the design and usability of the wearable and the station.

The Visual Behavior Monitor is a wearable device to continuously monitor visual behavior of the user. During the study, two functionally equivalent versions of the system were used, called Mark II and Mark III. The description below focuses on the latest system, Mark III.

The VBM is fixed on the spectacles frame with help of an adapter. Multiple adapters are provided to patients to allow attachment on all spectacles used by the patient. Patients can easily move the device from the adapter on one spectacle to the adapter on another when changing spectacles. The VBM is configured to start and stop measurements automatically when attached and detached from the adapter, as well as when spectacles are taken off.

After the VBM measurement was carried out, the respondent was asked the following questions: 1. Is it easy to attach the magnetic clip to the spectacles frames? 2. Is it easy is to attach the wearable 
to the magnetic clip? 3. Do you always feel comfortable using the wearable? 4 . Is it easy to use the wearable?

Regarding the station, the following questions were asked. 5. Is the connection between the station and the wearable always working? 6 . Is the information provided on screen always clear to you?

VBM (Figure 1) incorporates the following sensors: Two optical time-of-flight distance sensors directed forward and $30^{\circ}$ downward, ambient light sensor with separate red, green, and blue channels directed forward, combined ultraviolet and ambient light sensor directed upward, as well as motion and orientation sensors: Accelerometer, gyroscope, and magnetometer. The VBM does not include imaging sensors, such as cameras and location sensors, and does not infringe on the privacy of the wearer and other people. The VBM features an energy-independent real-time clock for reliable timestamping of the measurements.

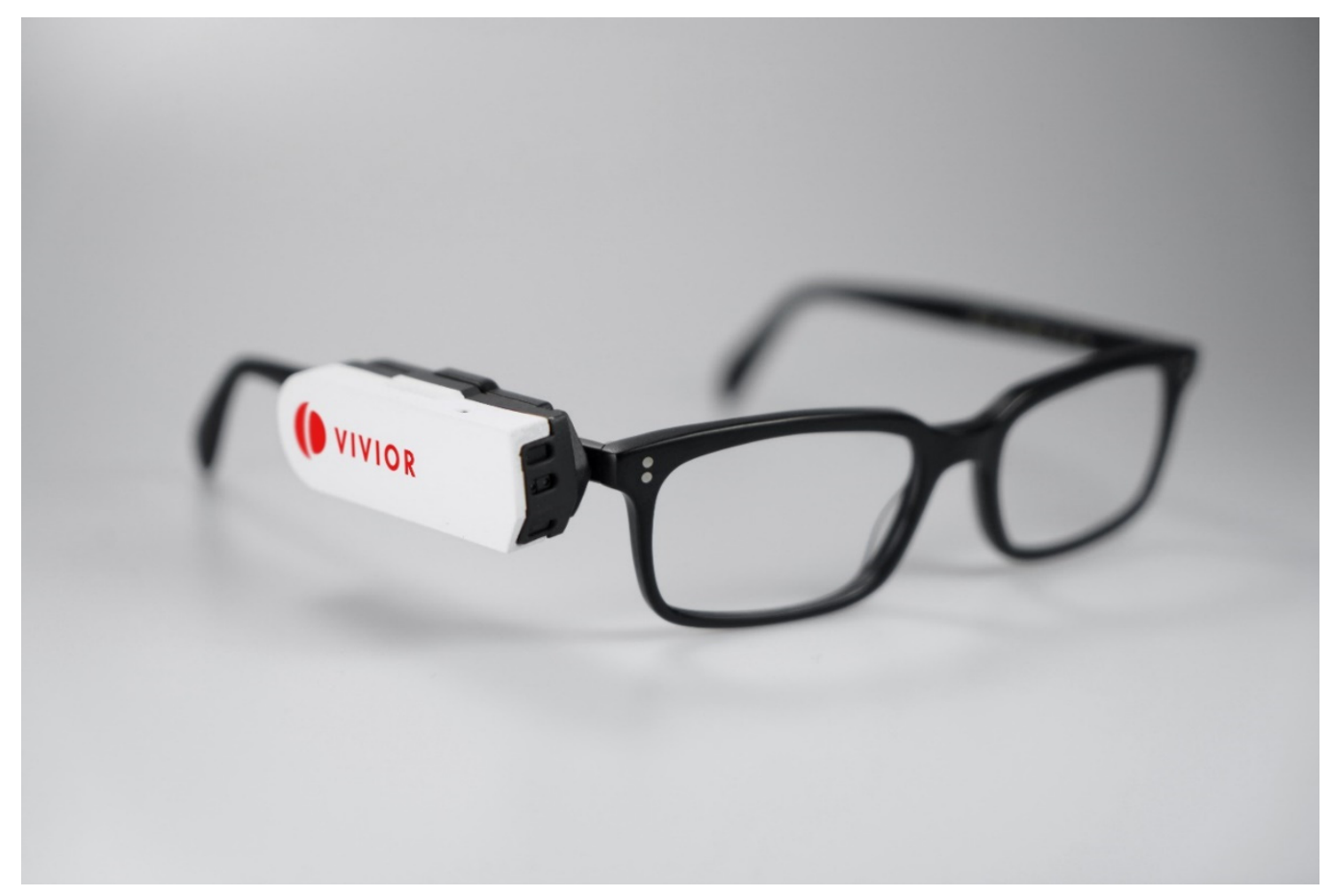

Figure 1. Visual Behavior Monitor (VBM) fixed on the spectacles.

Measurements data are stored in the encrypted format in the device and the progress of data collection is shown on the embedded screen or on the screen of the station in hours and minutes. The onboard memory capacity is more than $1000 \mathrm{~h}$. The VBM is charged overnight and the battery capacity is sufficient for a complete day of continuous measurements. When the device is returned to the clinic, data are transferred from the device to an electronic tablet and then uploaded to the internet server (cloud), decrypted, processed, and visualized via web-interface.

Cloud processing is performed by advanced machine learning algorithms, which are trained to recognize various types of patient's visual activities, such as looking on a desktop computer screen, reading handheld materials, driving a car, and viewing objects at a distance. The environment is further analyzed based on the recognized activities to derive relevant metrics such as viewing distances to the objects of visual activities, such as distance to a computer screen, to a desktop, or to handheld material. The data are further aggregated to create relevant statistical representations.

The VBM is intended to provide behavioral information to the attending HCP and to support ophthalmic surgeons or other healthcare professionals in the planning of cataract and refractive surgery. This is expected to improve participant satisfaction through the objective evaluation of individual vision needs and allowing practitioners to more adequately address those needs. Based on the individual 
profile of viewing distances, it is possible to select the optimal IOL solution by overlaying the defocus curve of the IOL, representing the expected visual acuity as a function of defocus (visual distance). Additional measured parameters, such as typical light levels and their spectral content, can be also considered. For example, monofocal IOLs deliver good far vision but cannot provide clear vision in near and intermediate distance zones, while lenses with advanced optics, such as bifocal and trifocal (multifocal) lenses, as well as lenses with an extended depth of focus, are capable of providing sufficient visual acuity and spectacle independence both in the near and intermediate zones, depending on the lens. At the same time, advanced optics can cause halos and glare in low-light conditions. Thus, for a patient with far distance vision needs with a large share of low-light activities, such as night driving, it would be reasonable to recommend a monofocal lens or blended vision solution, while an advanced optics IOL would be preferable for the patient with a range of activities with the viewing distance distributed between all vision zones and requiring often switching between zones.

The information provided by the VBM allows healthcare professionals to objectively assess the needs of the patient and explain the benefits and limitations of the available solutions to the patient. However, the decision should include other factors, like personal preferences of the patient and tolerance to the multifocality of IOLs with advanced optics, as well as other conditions and, thus, should not exclude discussion with the patient. The measuring elements used in the VBM are standard components. Furthermore, the VBM is not intended to replace state-of-the art diagnostic methods in refractive treatment. Nevertheless, it is very important to have hard facts to support the choice of IOL suitability or surgical method.

Patients use the VBM in their normal daily routine, with the only excluded activities being those that involve water exposure of the wearable. Some other activities may be exempt based on a benefit/risk assessment. Those activities are meant to be communicated verbally to the attending HCP during pre-operative interview.

A dedicated tablet is provided to the clinics for measurement data upload on the cloud and data review. The embedded cellular data module of the tablet is used for data upload and report review on the cloud, independent of the clinic's infrastructure.

The clinic has access to a detailed report of all data the VBM collects. A simplified patient report including recommendations is available for the patient.

The healthcare professional has an option to review aggregated metrics of visual behavior of the patient. For example, the viewing distance from all measurement days, collected by the patient, can be displayed as a histogram of the additional optical power (refraction) of the eye required to accommodate the objects of visual activity (Figure 2).

Further, it is possible to relate the viewing distances to the particular head inclinations in order to visualize viewing distances in a two-dimensional plane (Figure 3). Such visualization is helpful to educate patients on the relation of activities and visual distances as visual zones can be linked to the actual physical space.

Additionally, the system allows us to review activities chronologically, as performed by the patient during the selected measurement day. The distribution of viewing distances can be overlaid with other relevant parameters, such as ambient light exposure (Figure 4). 


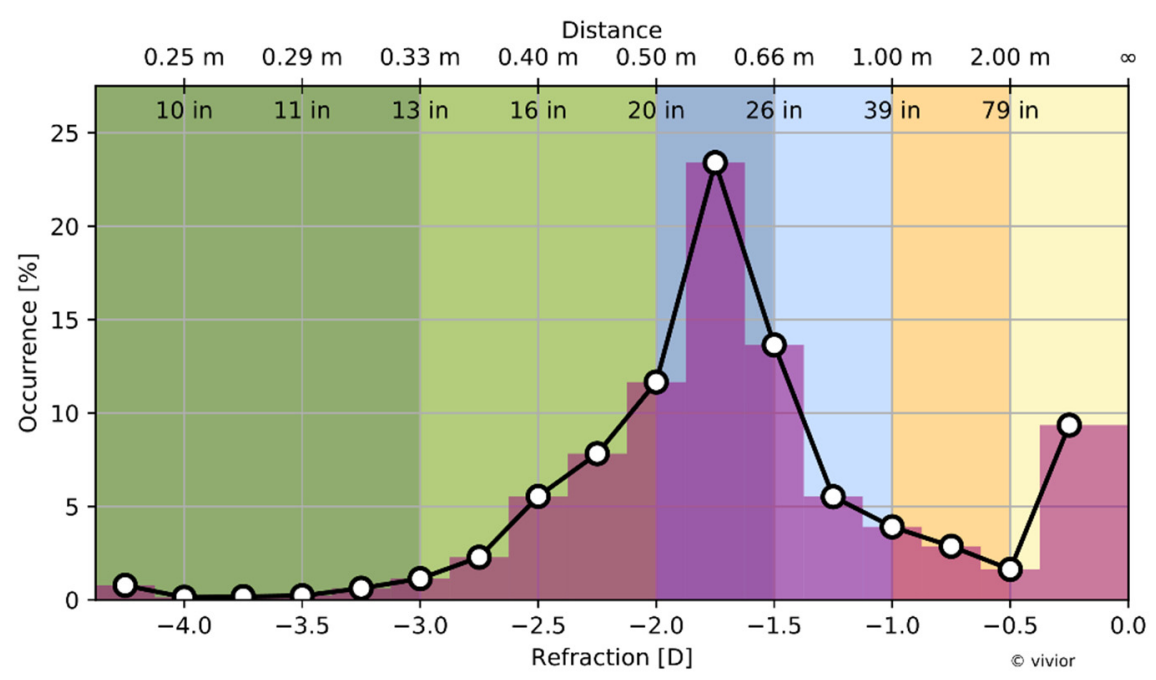

Figure 2. Distribution of viewing distance and required additional optical power of the eye (refraction) to observe objects of visual activity. The plot demonstrates domination of the intermediate viewing distance related to the computer work at around $-1.75 \mathrm{D}(0.57 \mathrm{~m})$ for the particular patient (Patient A). Color coding is used to display the viewing zone, with green representing near viewing distances, blue representing intermediate, and yellow representing far.

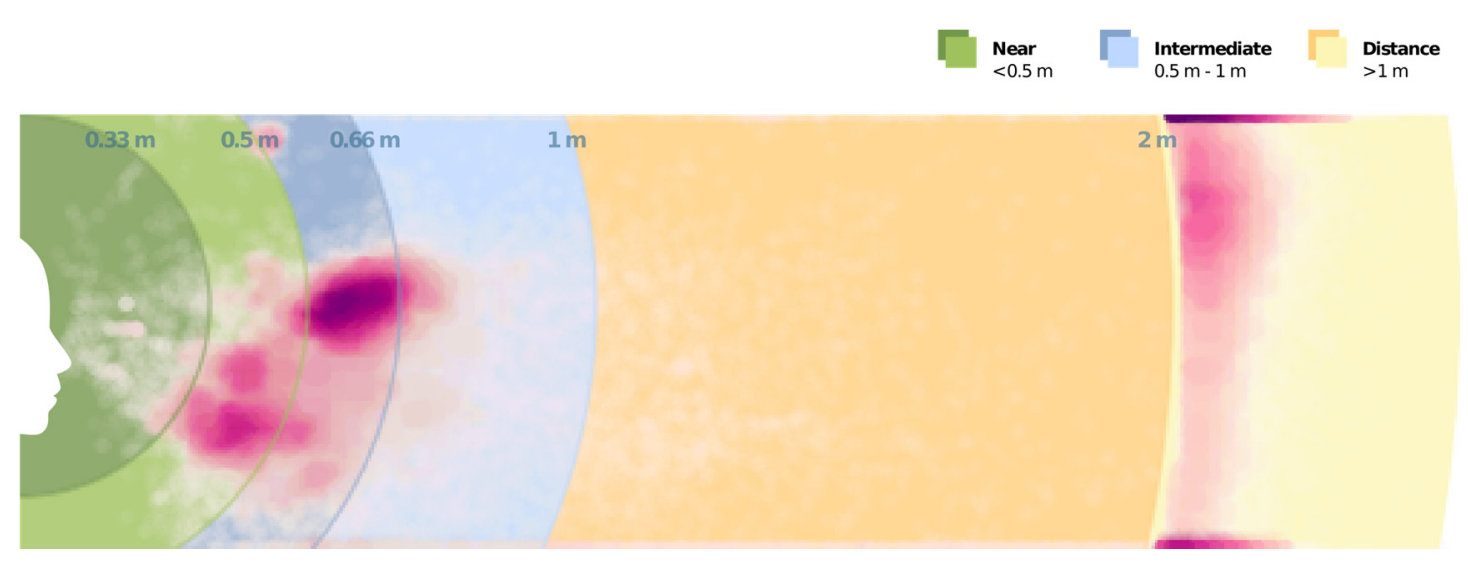

Figure 3. Mapping of viewing distances on two-dimensional plane using head flexion information (Patient A). Dense areas reflect the computer work and handheld devices. Color coding for the vision zones is the same as in Figure 2.

This kind of plot allows the demonstration of viewing conditions during various activities to the patient. The time of the day information helps the patient to relate his or her daily routine to the measurements and communicate to the HCP the importance of certain activities. The HCP is also able to relate viewing distance to other parameters. Figure 5 demonstrates the history of ultraviolet light exposure during the study day together with ambient light, which provides additional information about the patient's lifestyle.

The study has been conducted by five sites in three European countries: Germany, Switzerland, and Ireland (Table 1). A total of 129 patients were enrolled between August 2018 and May 2019. Patient age ranged from 49 to 82 (mean: 65). In all cases, the VBM measurement was performed before cataract surgery. 

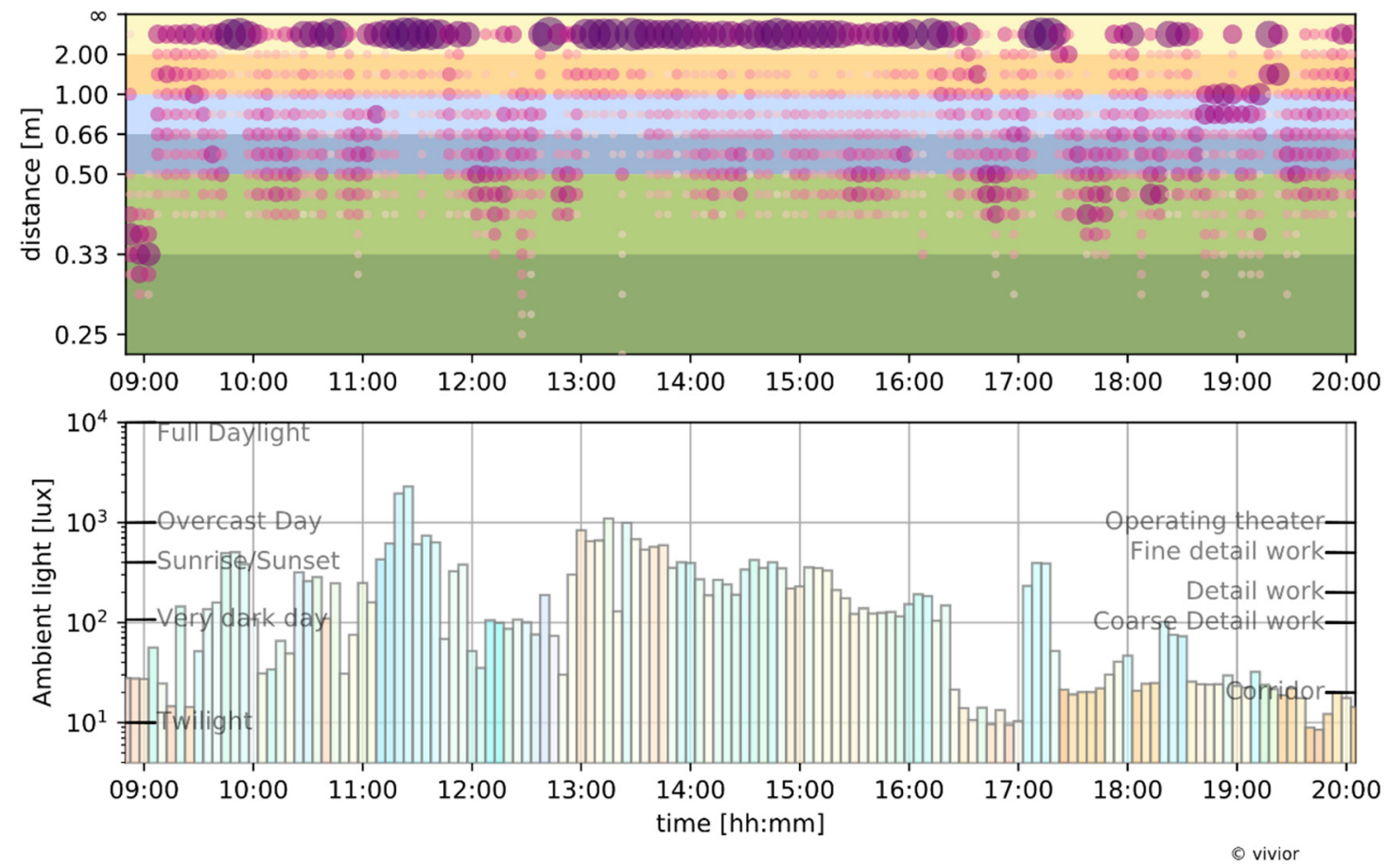

Figure 4. Example of the viewing distances and ambient light measurements with VBM during a day (Patient B). The example demonstrates the clear domination of far distances (upper plot), which is a marked difference in visual behavior from Patient A.

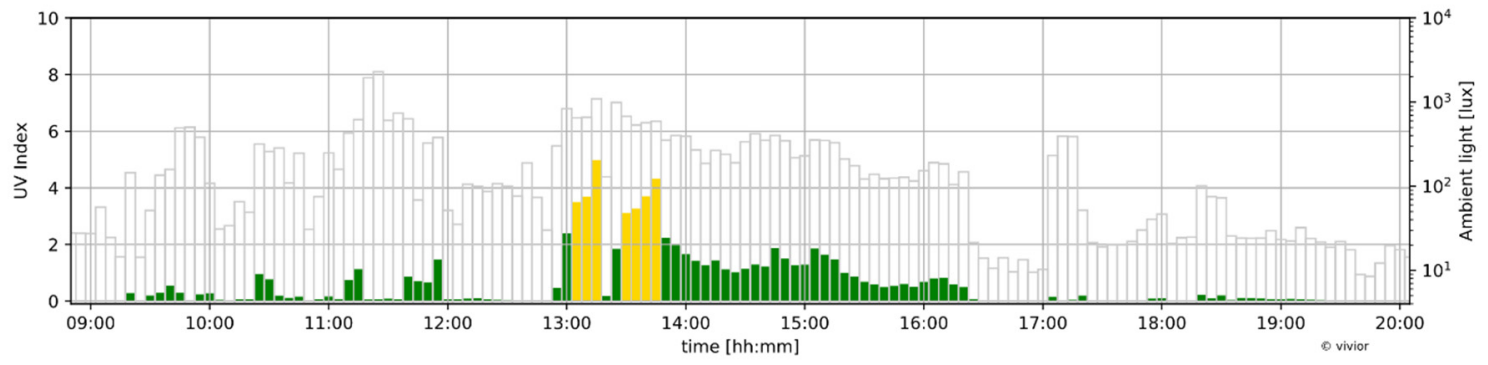

Figure 5. Example of the ultraviolet light exposure (quantified with UV index) as solid colored bars compared to ambient light level shown as empty bars during a measurement day shown in Figure 4 (Patient B). The level of ultraviolet light indicates that the example day was mostly spent outdoors.

Table 1. Vivior feasibility study sites, ethics committees, and enrollment.

\begin{tabular}{|c|c|c|}
\hline Study Center & Patients Enrolled & Ethics Committee Approval \\
\hline Wellington Eye Clinic, Dublin Ireland & 35 & $\begin{array}{l}\text { Beacon Hospital Research Ethics } \\
\text { Committee: BEA0096 }\end{array}$ \\
\hline $\begin{array}{l}\text { University Eye Clinic Bochum-Langendreer, } \\
\text { Bochum, Germany }\end{array}$ & 40 & $\begin{array}{c}\text { Ruhr University Ethics } \\
\text { Commission of Medical Faculty: } \\
\text { CIV-18-05-023986 }\end{array}$ \\
\hline Sehkraft Augenzentrum Köln Germany & 3 & $\begin{array}{c}\text { Ruhr University Ethics } \\
\text { Commission of Medical Faculty: } \\
\text { CIV-18-05-023986 }\end{array}$ \\
\hline Orasis Eye Clinic, Reinach, Switzerland & 40 & $\begin{array}{l}\text { Swiss Ethics: Ethikkommission } \\
\text { Nordwest- und } \\
\text { Zentralschweiz: 2018-01344 }\end{array}$ \\
\hline LASER VISTA, Basel Switzerland & 11 & $\begin{array}{l}\text { Swiss Ethics: Ethikkommission } \\
\text { Nordwest- und } \\
\text { Zentralschweiz: 2018-01344 }\end{array}$ \\
\hline Total & 129 & \\
\hline
\end{tabular}


All subjects gave their informed consent for inclusion before they participated in the study. The patients were informed in great detail about the content of the work, its usefulness, and its feasibility before the start of the study. The study was conducted in accordance with the Declaration of Helsinki, and the protocol was approved by the appropriate ethics committees for each site (Table 1).

\section{Results}

Of the total 178 eyes implanted, 119 eyes were implanted with monofocal intraocular lenses (67\%), 39 eyes were implanted with trifocals or multifocals IOLs (22\%), 15 eyes were implanted with toric lenses $(8 \%)$, and the remaining five eyes were implanted with lenses that were not identified by the surgeon (3\%). From these 129 patients included in the study, 178 cataract surgeries ultimately resulted, i.e., both eyes were not operated on in all cases, but only those that had an indication for surgery.

The pre-operative cataract scores distribution is shown in Figure 6 and the Cat-Quest 9 Task results are shown in Figure 7. A grade 3 or greater cataract manifested itself in $34 \%$ of the eyes, while no cataract and no reported cataract were accounted for in $29 \%$ of the eyes. The cataract and presbyopia limitations were also reflected in the pre-operative vision questionnaire with the vast majority of patients reporting some to very great difficulties in mastering everyday life and the majority of the patients being dissatisfied with the current eyesight.

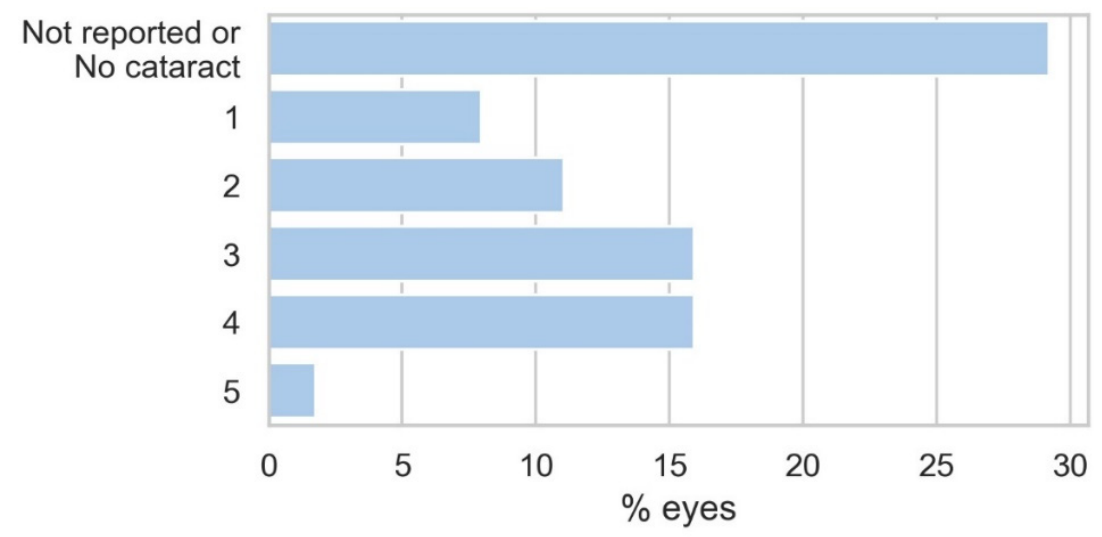

Figure 6. Lens opacities classification of cataract study patients.

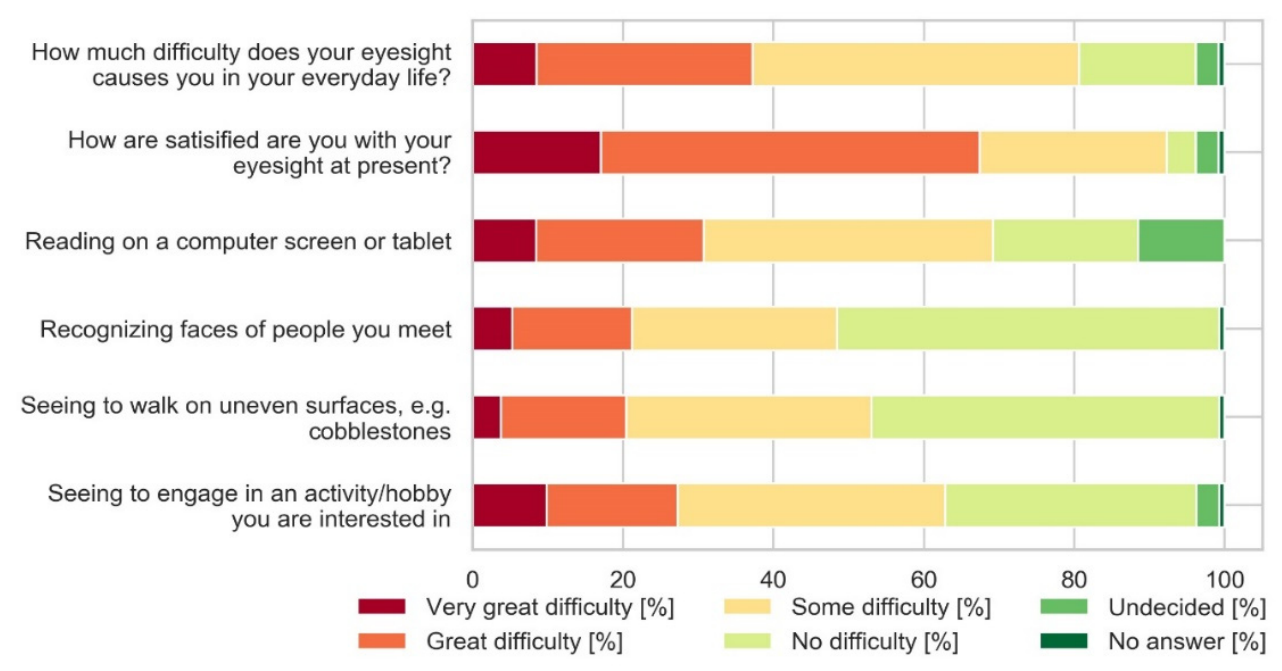

Figure 7. Pre-operative Cat-Quest 9 task results.

We have also investigated whether the difficulties in using the VMB system are related to the patients' visual problems. Based on the patients' answers to the usability questionnaire, the data were 
statistically evaluated using the parameters of corrected distant visual acuity and the Lens Opacities Classification System (LOCS) score. These results are shown in Table 2.

Table 2. Distribution of the preoperative corrected distance visual acuity and Lens Opacities Classification System (LOCS) III score of the patients separated by the answers in the usability questionnaire.

\begin{tabular}{|c|c|c|c|c|c|c|}
\hline \multirow{2}{*}{ Question } & \multicolumn{3}{|c|}{ Yes } & \multicolumn{3}{|c|}{ No } \\
\hline & $\mathbf{N}$ & $\begin{array}{c}\text { LOCS III } \\
\text { Score } \\
\text { Mean (Std) }\end{array}$ & $\begin{array}{c}\text { CDVA } \\
\text { logMAR } \\
\text { Mean (Std) }\end{array}$ & $\mathbf{N}$ & $\begin{array}{c}\text { LOCS III } \\
\text { Score } \\
\text { Mean (Std) }\end{array}$ & $\begin{array}{c}\text { CDVA } \\
\text { logMAR } \\
\text { Mean (Std) }\end{array}$ \\
\hline $\begin{array}{l}\text { 1. Is it easy to attach the magnetic clip to } \\
\text { your frames? }\end{array}$ & 113 & $2.087(1.179)$ & $0.209(0.215)$ & 10 & $1.500(1.323)$ & $0.118(0.161)$ \\
\hline $\begin{array}{l}\text { 2. Is it easy to attach the wearable to the } \\
\text { magnetic clip? }\end{array}$ & 118 & $2.093(1.178)$ & $0.206(0.213)$ & 5 & $0.625(0.750)$ & $0.111(0.197)$ \\
\hline $\begin{array}{l}\text { 3. Do you always feel comfortable using } \\
\text { the wearable? }\end{array}$ & 98 & $2.203(1.178)$ & $0.233(0.219)$ & 25 & $1.333(1.017)$ & $0.080(0.124)$ \\
\hline 4. Is it easy to use the wearable? & 111 & $2.053(1.186)$ & $0.208(0.217)$ & 11 & $1.889(1.364)$ & $0.148(0.161)$ \\
\hline $\begin{array}{l}\text { 5. Is the connection between the station } \\
\text { and wearable always working? }\end{array}$ & 82 & $2.175(1.178)$ & $0.221(0.209)$ & 22 & $1.525(1.282)$ & $0.192(0.220)$ \\
\hline $\begin{array}{l}\text { 6. Is the information provided on the } \\
\text { screen always clear to you? }\end{array}$ & 71 & $1.885(1.117)$ & $0.172(0.189)$ & 44 & $2.188(1.353)$ & $0.236(0.222)$ \\
\hline
\end{tabular}

Surprisingly, the groups that had difficulties with the VBM presented the higher visual acuity (lower logMAR values), as well as lower cataract scores (better vision), for all the questions, except for one concerning "information provided on the screen." The study included 15 patients with both eyes cataract-free and a relatively high distance-corrected visual acuity preparing for the clear lens exchange. This group was typically more demanding toward the usability of the system and often appeared in the group answering "no" in the questionnaire in Figure 8. This resulted in the proportion of patients without cataract in the "no" group being between $20 \%$ and $40 \%$, while the mean was around $12 \%$. The exception was question 6 where there was only a slightly higher proportion of clear lens patients in the "no" group (16\% vs. $11 \%$ ). Based on this data, we were not able to statistically attribute the difficulties reported in the usability questionnaire to the vision deficiencies of the patients for all questions except question 6, which also reported the highest negative feedback (34\% not agreeing that the information provided on the screen is always clear).

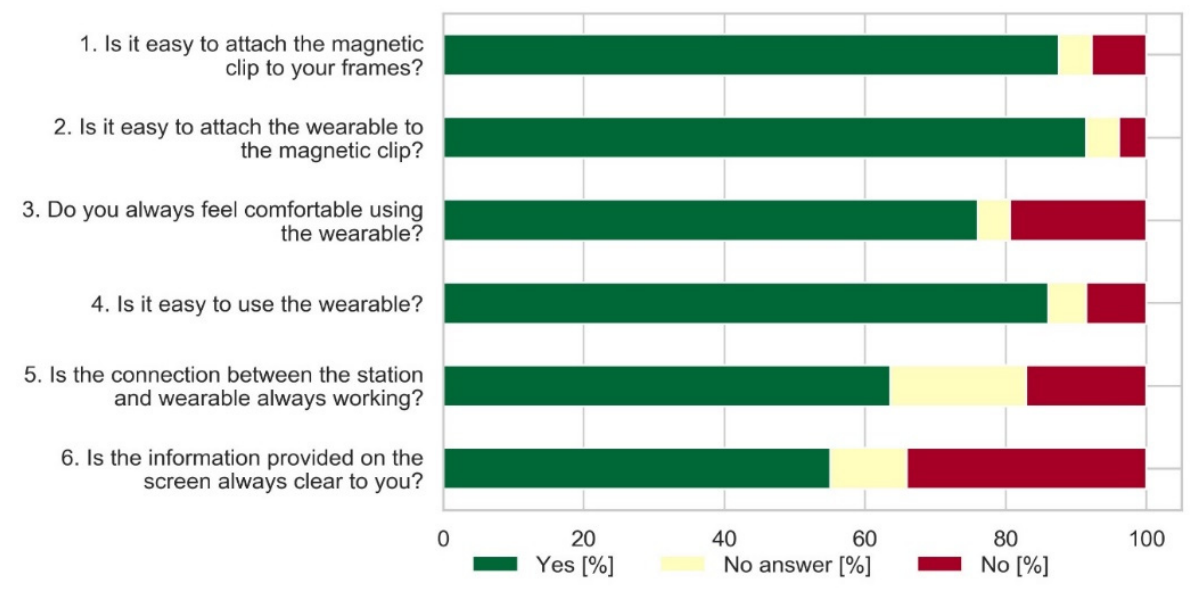

Figure 8. Results summary of usability questionnaire.

We have also studied the patient satisfaction with the vision results (Figure 9). 


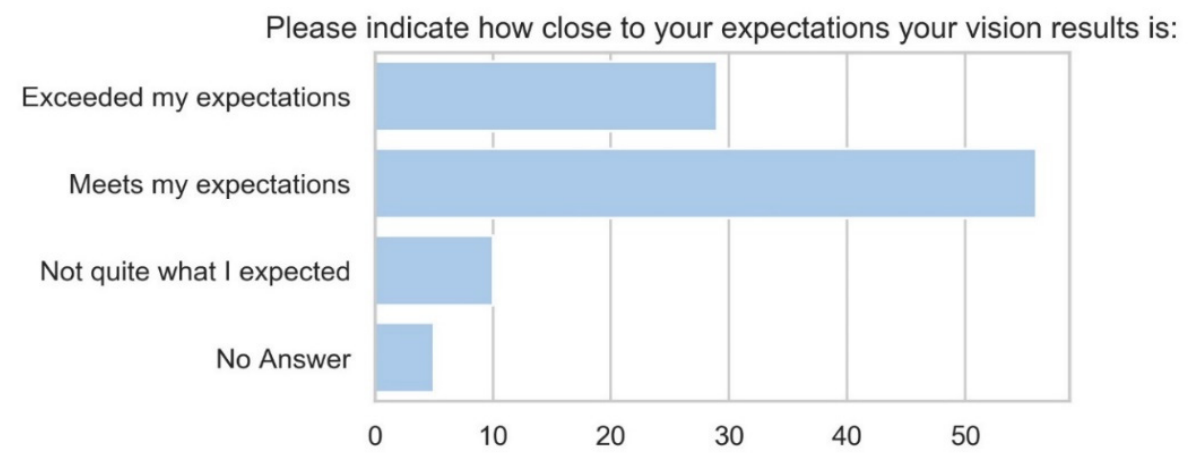

Figure 9. Post-operative Cat-Quest 9 results showing that surgical results met or exceeded patients' expectations.

A high level of patient satisfaction was reported, with $84 \%$ of patients meeting or even exceeding expectations (Figure 9).

\section{Discussion}

In today's world, visual acuity is not only becoming more and more important, but the demands on the visual system have increased significantly, whether at work or in leisure time. Various studies have shown that $61 \%$ of the patients prefer an explicit decision by the physician regarding the cataract surgery $[21,22]$. This strong dependency and importance of the physician's decision-making must be compared to the importance of patient involvement in order to maintain high patient satisfaction [23,24]. Previous studies show that the improving patients' perceived level of understanding is an important factor for the high level of patient satisfaction with the results of a cataract surgery [1,25-27]. There is an increasing demand to understand patient needs and expectations when planning cataract and refractive surgeries. It is generally accepted that understanding patient behavior and expectations and including this information in the planning process results in better surgical outcomes. Psychometric tests in the form of cataract surgery outcome questionnaires have been developed, identifying the limitations of performing daily tasks due to an individual's vision, a trait known as visual functioning $[15,16]$. The use of questionnaires and visual assessments in measuring patient outcomes is effective; however, the subjectivity of these methods is questionable. Nevertheless, questionnaires are very important in addition to objective measurement, because they reflect the important subjective part of the patient's feelings. Its importance and introduction have been used in the literature for a long time where specific questionnaires have been developed for specific questions [23], as we have used it in our study. Current psychometric measures may be subject to systematic error such as recall bias and could cause judgment errors resulting in patient discontent with surgical outcomes $[19,20]$. Integrating technology, such as the wearable VBM, which collects objective visual behavior data for pre-operative patient assessments, has shown improvement in the current understanding of patient visual behavior. It was very gratifying to note that the application of the VBM was, in the vast majority of cases, problem-free. Thus, the VBM measuring device could be installed with reasonable effort and be reliably used by the patient. In this sense, the goal was achieved to design a VBM in a way that not only works reliably but is also easy to use. Most of the study patients stated that they had severe to very severe visual problems before the surgery. None of the patients who reported very great difficulties in their daily tasks reported troubles in attaching the clip to the spectacles frame or VBM to the clip. The results of the analysis of the patients' visual performance based on their responses to the questionnaire showed that the group that was not satisfied with the user-friendliness had, on average, a higher visual acuity and a lower LOCS score, with the exception of one question related to the presentation of the information on the screen. This effect of the higher reported usability problems of patients with better vision can be attributed to the higher demands of patients with clear natural lenses. The feedback from patients on the system handling was mostly related to the adaptation to a specific spectacle frame, the correct 
alignment of the VBM and clip, and the strength of the magnetic attachment. This feedback has been considered in the development of the next generation of the system. The size of the clip has been adapted to make it easier to handle, and the shape of the clip and wearable has been redesigned to visually match each other to make the attachment more intuitive. The clip has a defined arrow shape that points forward to indicate the direction of attachment. The magnetic attachment has been replaced by a purely mechanical attachment to prevent the design from falling off during heavy movement, while at the same time being easy to attach and detach. The reported difficulties in understanding the information on the station's screen and connection problems between the wearable and the station have led to the decision to make the next unit self-sufficient and to omit an additional station for data acquisition, thus eliminating both station-related difficulties.

The objective data generated could aid clinicians in providing a highly individualized evaluation of a patient's visual needs, including estimated viewing distances, and determine the right course of action to improve post-operative patient outcomes and patient satisfaction. The subjective and objective data of patients often coincide. However, the patient's subjective feelings are not always correctly interpreted by them. The objective data collected can close this circle to a higher percentage and thus have potential to increase patient satisfaction. Including objective data of the visual behavior of each individual patient is another important step for a successful customized treatment.

Prior to cataract- and refractive surgery in addition to a comprehensive eye exam in terms of the overall health of the eyes, refraction measurements (amount of nearsightedness, farsightedness, astigmatism), determining the corneal curvature, as well as the length of the eye, it is particularly important to analyze the environmental conditions in the eye and ophthalmological concomitant diseases. The objectively measured ophthalmological findings, as well as the VBM data collected, have the potential to further improve treatment outcomes.

There is no ideal correction for all distances currently available, but the objective measurements of viewing distances, ambient light conditions, and head inclinations with devices such as the VBM will potentially allow better characterization of a patient's lifestyle and support the choice of IOL solutions better matching a specific patient's lifestyle and visual needs. Further studies with extended questions are ongoing.

\section{Conclusions}

To test the VBM device usability among patients, a multi-center feasibility study was conducted. Patients found the device easy to use, with most reporting that the device was not intrusive. The patients, even those that were elderly with significant cataracts, were able to comfortably use the VBM. Hence, the results of this feasibility study demonstrated that the concept of wearable monitoring of visual behavior is, in general, accepted by the patients. VBM may be used correctly and reliably by the patient. The difficulties of handling the system by the patients have been considered in the design of the next generation of the VBM.

The objective data collected with the VBM may have the potential to improve treatment outcomes, and the next research phase will seek to objectively assess the impact of the VBM on surgeons' treatment decision making.

Author Contributions: B.P. provided the treatment indication, developed the study design, acquired clinical data, and contributed to writing the paper. Z.C. contributed substantially to the methodology and substantially contributed to writing the paper. B.P.-E. performed substantial data curation. P.Z. performed data analysis and contributed to the study design. All authors have read and agreed to the published version of the manuscript.

Funding: This research received no external funding.

Conflicts of Interest: Bojan Pajic, Brigitte Pajic-Eggspuehler, and Zeljka Cvejic declare no financial interest. It is to declare that Pavel Zakharov is an employee of Vivior AG. 


\section{References}

1. Pager, C.K. Expectations and outcomes in cataract surgery: A prospective test of 2 models of satisfaction. Arch. Ophthalmol. 2004, 122, 1788-1792. [CrossRef] [PubMed]

2. Wang, W.; Yan, W.; Fotis, K.; Prasad, N.M.; Lansingh, V.C.; Taylor, H.R.; Finger, R.P.; Facciolo, D.; He, M. Cataract Surgical Rate and Socioeconomics: A Global Study. Investig. Ophthalmol. Vis. Sci. 2016, 57, 5872-5881. [CrossRef] [PubMed]

3. Aristodemou, P.; Sparrow, J.M.; Kaye, S. Evaluating Refractive Outcomes after Cataract Surgery. Ophthalmology 2019, 126, 13-18. [CrossRef] [PubMed]

4. Pajic, B.; Massa, H.; Eskina, E. Presbyopiekorrektur mittels Lasechirurgie. Klin. Monatsbl. Augenheilkd. 2017, 234, e29-e42. [PubMed]

5. Knorz, M.C. Presbyopia Correction with Intraocular Lenses. Klin. Monbl. Augenheilkd. 2020, 237, $213-223$. [PubMed]

6. Goldberg, D.G.; Goldberg, M.H.; Shah, R.; Meagher, J.N.; Ailani, H. Pseudophakic mini-monovision: High patient satisfaction, reduced spectacle dependence, and low cost. BMC Ophthalmol. 2018, 18, 293. [CrossRef] [PubMed]

7. Tarib, I.; Kasier, I.; Herbers, C.; Hagen, P.; Breyer, D.; Kaymak, H.; Klabe, K.; Lucchesi, R.; Teisch, S.; Diakonis, V.F.; et al. Comparison of Visual Outcomes and Patient Satisfaction after Bilateral Implantation of an EDOF IOL and a Mix-and-Match Approach. J. Refract Surg. 2019, 35, 408-416. [CrossRef]

8. Hovanesian, J.A.; Lane, S.S.; Allen, Q.B.; Jones, M. Patient-Reported Outcomes/Satisfaction and Spectacle Independence with Blended or Bilateral Multifocal Intraocular Lenses in Cataract Surgery. Clin. Ophthalmol. 2019, 13, 2591-2598. [CrossRef]

9. Shih, W.; Liu, J. A calibration-free gaze tracking technique. In Proceedings of the 15th Conference Patterns Recognition, Barcelona, Spain, 3-7 September 2000; Volume 4, pp. 201-204.

10. Ji, Q.; Yang, X. Real-time eye, gaze and face pose tracking for monitoring driver vigilance. Real-Time Imaging 2002, 8, 357-377. [CrossRef]

11. Grace, R. Drowsy driver monitor and warning system. In Proceedings of the International Driving Symposium on Human Factors Driver Assessment, Training and Vehicle Design, Aspen, CO, USA, 14-17 August 2001; pp. 64-69.

12. Bergasa, L.M.; Nuevo, J.; Sotelo, M.A.; Barea, R.; Lopez, M.E. Real-Time System for Monitoring Driver Vigilance. In IEEE Transactions on Intelligent Transportation Systems; IEEE: New York, NY, USA, 2006; Volume 7.

13. Lodhia, V.; Karanja, S.; Lees, S.; Bastawrous, A. Acceptability, usability, and views on deployment of peek, a mobile phone mHealth intervention for eye care in Kenya: Qualitative study. JMIR mHealth uHealth 2016, 4, e30. [CrossRef]

14. Groessl, E.J.; Liu, L.; Sklar, M.; Tally, S.R.; Kaplan, R.M.; Ganiats, T.G. Measuring the impact of cataract surgery on generic and vision-specific quality of life. Qual. Life Res. 2013, 22, 1405-1414. [CrossRef] [PubMed]

15. Stelmack, J. Quality of life of low vision patients and outcomes of low vision rehabilitation. Optom. Vis. Sci. 2001, 78, 335-342. [CrossRef] [PubMed]

16. McAlinden, C.; Gothwal, V.K.; Khadka, J.; Wright, T.A.; Lamoureux, E.L.; Pesudovs, K. A head-to-head comparison of 16 cataract surgery outcome questionnaires. Ophthalmology 2001, 118, 2374-2381. [CrossRef] [PubMed]

17. Lundström, M.; Pesudovs, K. Catquest-9SF patient outcomes questionnaire. Nine-item short-form Rasch-scaled revision of the Catquest questionnaire. J. Cataract Refract. Surg. 2009, 35, 504-513. [CrossRef] [PubMed]

18. Morlock, R.; Wirth, R.J.; Tally, S.R.; Garufis, C.; Heichel, C.W.D. Patient-Reported Spectacle Independence Questionnaire (PRSIQ): Development and Validation. Am. J. Ophthalmol. 2017, 178, 101-113. [CrossRef]

19. Nogueira, H.N.; Alves, M.; Schor, P. Preoperative automatic visual behavioural analysis as a tool for intraocular lens choice in cataract surgery. Arq. Bras. Oftalmol. 2015, 78, 94-99. [CrossRef]

20. Gibbons, A.; Ali, T.K.; Waren, D.P.; Donaldson, K.E. Causes and correction of dissatisfaction after implantation of presbyopia-correcting intraocular lenses. Clin. Ophthalmol. 2016, 10, 1965-1970. [CrossRef]

21. Kiss, C.G.; Richter-Mueksch, S.; Stifter, E.; Diendorfer-Radner, G.; Velikay-Parel, M.; Radner, W. Informed consent and decision making by cataract patients. Arch. Ophthalmol. 2004, 122, 94-98. [CrossRef] 
22. Henderson, B.A.; Solomon, K.; Masket, S.; Potvin, R.; Holland, E.J.; Cionni, R.; Sandoval, H. A survey of potential and previous cataract-surgery patients: What the ophthalmologist should know. Clin. Ophthalmol. 2014, 25, 1595-1602.

23. Berg, A.; Yuval, D.; Ivancovsky, M.; Zalcberg, S.; Dubani, A.; Benbassat, J. Patient perception of involvement in medical care during labor and delivery. Israel Med. Assoc. J. 2001, 3, 352-356.

24. Janz, N.K.; Wren, P.A.; Copeland, L.A.; Lowery, J.C.; Goldfarb, S.L.; Wilkins, E.G. Patient-physician concordance: Preferences, perceptions, and factors influencing the breast cancer surgical decision. J. Clin. Oncol. 2004, 1, 3091-3098. [CrossRef] [PubMed]

25. Addisu, Z.; Solomon, B. Patients' preoperative expectation and outcome of cataract surgery at jimma university specialized hospital-department of ophthalmology. Ethiop. J. Health Sci. 2011, 21, 47-55. [CrossRef] [PubMed]

26. Chen, Z.; Lin, X.; Qu, B.; Gao, W.; Zuo, Y.; Peng, W.; Jin, L.; Yu, M.; Lamoureux, E. Preoperative Expectations and Postoperative Outcomes of Visual Functioning among Cataract Patients in Urban Southern China. PLoS ONE 2017, 9, e0169844. [CrossRef] [PubMed]

27. Leidy, N.K.; Beusterien, K.; Sullivan, E.; Richner, R.; Muni, N.I. Integrating the patient's perspective into device evaluation trials. Value Health 2006, 9, 394-401. [CrossRef] [PubMed]

(C) 2020 by the authors. Licensee MDPI, Basel, Switzerland. This article is an open access article distributed under the terms and conditions of the Creative Commons Attribution (CC BY) license (http://creativecommons.org/licenses/by/4.0/). 